\title{
Acute ST-segment elevation myocardial infarction in a young patient with essential thrombocythemia: a case with long-term follow-up report
}

\author{
This article was published in the following Dove Press journal: \\ Journal of Blood Medicine \\ 23 July 2014 \\ Number of times this article has been viewed
}

\author{
Tariq Bhat ${ }^{\prime}$ \\ Mohammed Ahmed ${ }^{2}$ \\ Hassan Baydoun ${ }^{2}$ \\ Zahraa Ghandour ${ }^{3}$ \\ Alina Bhat ${ }^{2}$ \\ Donald McCord' \\ 'Division of Cardiology, ${ }^{2}$ Department \\ of Medicine, Staten Island University \\ Hospital, Staten Island, NY, USA; \\ ${ }^{3}$ Department of Internal Medicine, \\ Faculty of Medical Sciences, Lebanese \\ University, Beirut, Lebanon
}

\begin{abstract}
Essential thrombocythemia (ET) is a neoplastic proliferation of mature myeloid cells - in particular, megakaryocytes - leading to persistently elevated platelet count. Usual clinical presentation is related to an increase in the risk of hemorrhage and/or thrombosis. Management of ET consists of antiplatelet therapies - mainly aspirin and cytoreductive therapies. Coronary involvement in patients with ET is rare. The optimal treatment strategies for ET patients presenting with acute myocardial infarction remains unclear. Acute interventions like intracoronary thrombolytic therapy, angioplasty, and coronary-artery bypass grafting have been reported in such patients. However, several questions remain unanswered about the acute and long-term management of these patients. Herein, we report the case of a 47-year-old female who presented with acute myocardial infarction as the first clinical sign of ET, and also present the long-term follow-up of this patient.
\end{abstract}

Keywords: STEMI, platelets, hypercoagulability, thrombosis, antiplatelets

\section{Introduction}

Essential thrombocythemia (ET) is a neoplastic proliferation of mature myeloid cells - in particular, megakaryocytes - leading to persistently elevated platelet count. It is an uncommon disease with an incidence of 2.5 per 1 million. ${ }^{1}$ Clinical symptoms are related to an increase in the risk of hemorrhage and/or thrombosis. Recent studies have demonstrated that thrombotic complications in the cerebral, coronary, and peripheral vessels are more common than hemorrhagic complication. ${ }^{1,2}$

ET is a disease that predominantly affects males and females of a median age of $60 .{ }^{3}$ Coronary involvement in patients with ET is rare; however, herein, we report the case of a 47-year-old female who presented with acute myocardial infarction (MI) as the first clinical sign of ET. We also report the long-term (3-year) follow-up of this same patient.

\section{Case presentation}

A 47-year-old female patient presented to our emergency department with chest pain. The pain was: retrosternal; of sudden onset, starting 2 hours prior to presentation at our hospital; continuous and worsening in severity; described as pressure; irradiating to the neck and left upper extremity; had no precipitating or alleviating factors; and was associated with nausea, diaphoresis, and shortness of breath. She denied headache, dizziness, syncope, palpitations, or vomiting. Prior to the pain beginning, the patient
Correspondence: Hassan Baydoun 475 Seaview Ave, Staten Island, NY 10305, USA

$\mathrm{Tel}+\mathrm{I} 7 \mid 82269000$

$\mathrm{Fax}+17182261983$

Email baydounhassan@hotmail.com 
denied having any of the symptoms. Review of her system was negative except for the symptoms mentioned.

The patient had no significant medical history except that she was seen many years prior for mild thrombocytosis during her pregnancy. At that time, she was started on aspirin. She did not keep her follow-up appointments afterward. Her surgical history was significant for hysterectomy.

Her father had had coronary artery disease and MI at the age of 50; he died at the age of 67. Her mother had been diagnosed with chronic myelogenous leukemia and succumbed to blastic crisis after 7 years. Three of the patient's four children were positive for Factor V Leiden and one daughter developed extensive deep-vein thrombosis after 4 weeks on the oral contraceptive pills and major bleeding on warfarin (severe hemorrhagic ovarian cysts).

The patient was a nonsmoker and denied any alcohol or drug use. She had no history of allergy. Her only medication was baby aspirin once daily.

On initial examination, the patient was awake, alert, and oriented. Her blood pressure was 90/60 $\mu \mathrm{g}$, and she had a pulse of 80 beats per minute, respiratory rate of 12 breaths per minute, and a temperature of $99^{\circ} \mathrm{F}$. There was no jugular vein distention. Auscultation was clear throughout both lungs. Cardiac auscultation revealed an S4 gallop. No loud murmurs were appreciated. Abdominal exam was unremarkable, with no hepatosplenomegaly. There was no edema of the lower extremities. There was positive erythromelalgia of the hands.
Her electrocardiogram showed sinus rhythm and STsegment elevation in the anterior leads from V1 to V6 consistent with a large anterior wall infarct (Figure 1).

Initial blood work showed negative cardiac enzymes. Her hemoglobin was $13.7 \mathrm{~g} / \mathrm{dL}$, white blood cell count was $14.2 \mathrm{th} / \mathrm{mm}^{3}$, and platelet count was $935,000 \mathrm{th} / \mathrm{mm}^{3}$. The patient's basic metabolic profile was normal. Her low-density lipoprotein and high-density lipoprotein were $148 \mathrm{mg} / \mathrm{dL}$ and $61 \mathrm{mg} / \mathrm{dL}$, respectively.

The patient was transferred urgently to the catheterization lab. Left heart catheterization showed a total proximal left anterior descending artery (LAD) occlusion (Figure 2A), while her other coronary arteries were normal. A successful balloon angioplasty, with two bare metal stents, was performed on the $100 \%$ lesion in the proximal LAD, and an excellent result was attained (Figure 2B). The patient was started, in addition to aspirin (325 mg daily), on clopidogrel, a statin, and beta-blockers. Transthoracic echocardiography revealed a left ventricular ejection fraction of $35 \%$ with severe hypokinesia of the mid-apical anteroseptal and apical walls.

During her hospital stay, the patient was seen by the hematology-oncology team. Further workup was done and this showed a positive $J A K-2$ mutation. She was negative for the Factor V Leiden mutation $(R 506 Q)$ and $B C R-A B L$ genes translocation (Philadelphia chromosome). The rest of her blood work was within normal limits, including the erythrocyte sedimentation rate $(1 \mathrm{~mm} / \mathrm{h})$, ferritin

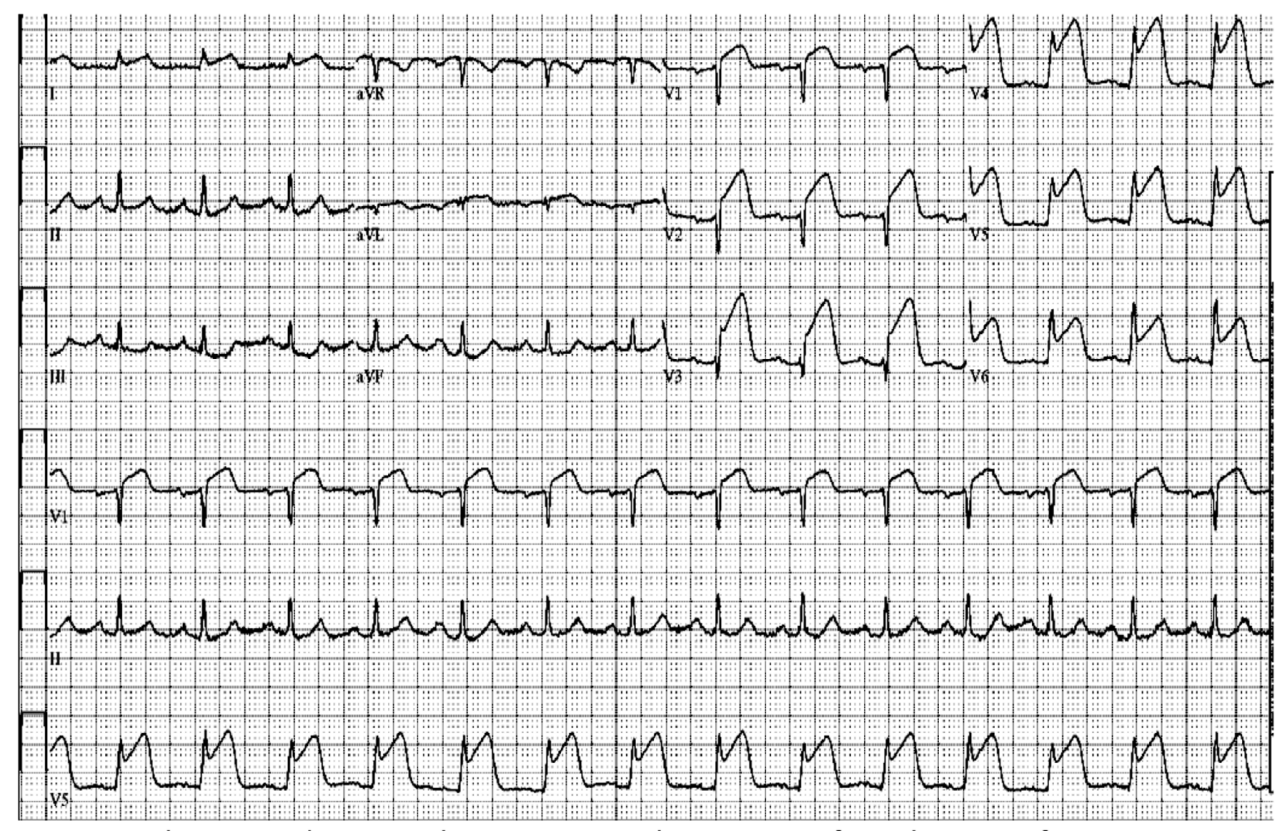

Figure I Electrocardiogram showing more than $2 \mathrm{~mm}$ of ST elevation from $\mathrm{VI}$ to $\mathrm{V} 6$. 


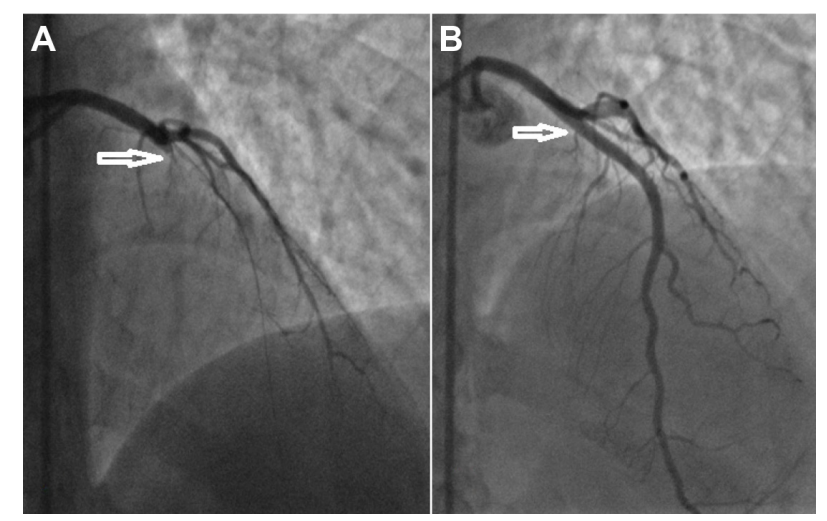

Figure 2 Left coronary angiography (right anterior oblique cranial). (A) Proximal total occlusion of the left anterior descending artery. (B) Successful angioplasty with two bare metal stents.

(151 ng/mL), thyroid-stimulating hormone $(1.3 \mathrm{mIU} / \mathrm{mL})$, folate $(10.2 \mathrm{ng} / \mathrm{mL})$, vitamin B12 (382 pg/mL) and bleeding time (1.1 min). Patient was diagnosed with ET and started on hydroxyurea 1,000 mg twice daily. Patient was discharged in a stable condition with the plan to follow up with the cardiologist and hematologist.

Two weeks later, during a follow-up at the hematology office, her complete blood count revealed a platelet count of $803 \mathrm{th} / \mathrm{mm}^{3}$, so the dose of hydroxyurea was increased to $2,500 \mathrm{mg}$ twice daily. Subsequent follow-up a few weeks later indicated a platelet count of $310 \mathrm{th} / \mathrm{mm}^{3}$, so the patient was kept on the same dose for the next 3 years. Her platelet count four months ago was still within the normal range.

The patient has been followed-up in the outpatient cardiology office for the last 3 years. Within a few months, her left ventricular function was found to have improved to normal and has been normal since then. We managed her as a coronary artery disease (CAD) equivalent, continued on dual antiplatelet therapy (aspirin and clopidogrel) beyond 1 year.

\section{Discussion}

ET is a myeloproliferative disorder characterized by abnormal proliferation of megakaryocytes, and it causes thrombus formation in the systemic arteries including the coronary arteries. Coronary artery involvement leading to acute MI is rare compared with other myeloproliferative diseases like polycythemia vera (PV). ${ }^{4}$ Rossi et al followed 170 patients with ET for 10 years. ${ }^{4}$ Only $9.4 \%$ of these patients had an MI during the follow-up period, and, of those, $75 \%$ had additional cardiovascular risk factors. There are many theories about the factors contributing to MI in ET patients. However, the underlying pathophysiological mechanisms still remain unclear. It has been reported that hypertension and cigarette smoking are risk factors for thrombosis development in patients with ET. ${ }^{5}$ Except for the family history of coronary artery disease, our patient, a nonsmoker, had no other traditional risk factors for MI, such as dyslipidemia, diabetes mellitus, or hypertension. According to her profile (age $<60$ years, no history of thrombosis, and platelet count $<1$ million $/ \mu \mathrm{L}$ ), the patient is stratified as low risk for thrombosis and hemorrhage. When MI occurs in a patient who does not have these traditional risk factors, then alternative etiologies should be investigated. They include hypercoagulable states, coronary vasospasm, coronary inflammation, anomalous coronary arteries, coronary dissection, and embolization. ${ }^{6}$ Previous investigations for the pathogenesis of acute coronary syndrome (ACS) have shown the importance of platelet thrombus formation caused by vascular endothelial damage resulting from plaque rupture. ${ }^{7}$ However, thrombus formation due to abnormal platelet count might be the main underlying cause of ACS associated with ET. Douste-Blazy et al reported that, in cases of ET, the five pathophysiologic mechanisms of thrombus formation in different coronary arteries were: 1) activation of thrombocytes as a result of endothelial damage, 2) extended arterial spasms resulting in formation of thrombus, 3 ) increased procoagulant activity of thrombocytes, 4) changes in glycoproteins on the surface of thrombocytes, and 5) possible deficiency of selective lipoxygenase. ${ }^{8}$

Hypercoagulable states are associated with myeloproliferative disorders such as PV, chronic myelogenous leukemia, ET, and primary myelofibrosis. Patients with myeloproliferative disorders have an elevated risk of both hemorrhage and thrombosis. Thrombotic complications are more prevalent than hemorrhagic complications. ${ }^{9}$ Thrombotic occlusions occur more frequently in the arteries than veins; further, strokes are the most frequent presentation, followed by MI and peripheral arterial occlusion. ${ }^{6}$

In ET, megakaryocytes exhibit an increased sensitivity to thrombopoietin. ${ }^{10}$ An important question to ask at this point is what are the central molecular mechanisms that underlie the activation of the coagulation system and lead to thrombosis? Janus kinase (JAK) is an intracellular protein kinase. Phosphorylation of JAK activates cytoplasmic signal transducers and activators of transcription (STAT). ${ }^{11}$ The STAT proteins then shuttle into the nucleus and activate the downstream pathways in thrombopoietin and erythropoietin signaling, as well as activating receptors for cytokines, colony stimulating factors, and interferons. ${ }^{6,11}$ The JAK2 V615 mutation, an acquired somatic mutation, results in a mutated tyrosine kinase that continuously activates the STAT proteins, which, in turn, activate the downstream pathways 
discussed. The presence of a JAK2 mutation has become a major diagnostic criterion for PV, ET, and myelofibrosis. ${ }^{6}$

Management of ET consists of antiplatelet therapies mainly aspirin and cytoreductive therapies that consist of phlebotomy, hydroxyurea, anagrelide, interferon- $\alpha$, and ruxolitinib, which is an inhibitor of JAK 1 and JAK2 (and recently approved by the US Food and Drug Administration). ${ }^{6}$ Aspirin combined with cytoreductive therapy is thought to be highly effective in PV and ET patients. ${ }^{3}$ Antiplatelet therapy should be used with caution and its benefits weighed against the risks of serious bleeding, especially in patients with a platelet count of $1,000 \times 10 /$ L $^{10,12}$ Cytoreductive therapies are considered in patients with myeloproliferative disorders who are over the age of 60 , at high risk for thrombotic events, have a significant history of thrombotic or hemorrhagic events, and a platelet count $>1.5$ million $/ \mathrm{mm}^{3} .{ }^{3}$ Cytoreductive therapies must also be weighed against the risk of leukemic transformation.

The optimal treatment strategies for ET patients presenting with acute MI remain unclear. Acute interventions like intracoronary thrombolytic therapy, angioplasty, and coronary-artery bypass grafting have been reported in such patients. ${ }^{13}$ Intracoronary thrombus remains a challenge for interventional catheter-based techniques, and distal embolization is considered to be a major cause of insufficient reperfusion. ${ }^{14}$ A previous report showed that the use of both aspiration thrombectomy and distal protection to prevent distal embolization achieved a successful angioplasty without complications. ${ }^{15}$ Mizuta et al reported a case of acute MI in a patient with ET treated with percutaneous transluminal coronary recanalization by administration of urokinase that resulted in successful revascularization with thrombolysis in myocardial infarction (TIMI) grade III coronary flow. ${ }^{5}$

However, there is no clear evidence on how to manage these patients on a long-term basis and whether we should treat them as atherosclerotic CAD equivalent. A previous study showed that a history of thrombosis at diagnosis was significantly associated with recurrent thrombosis. ${ }^{16}$ Further, in a review by Landolfi and Di Gennaro, the authors considered that more aggressive antiplatelet therapy with a combination of aspirin and clopidogrel in patients with ACS, like our patient, might be beneficial for preventing rethrombosis. ${ }^{17}$ In our case, the patient had no risk factors for CAD except family history, and we knew that the etiology of her MI was thrombotic process; despite that, we managed her as per American College of Cardiology/American Heart Association guidelines ${ }^{18}$ for CAD. Our patient has done well over the last 3 years, never complaining of any symptoms or acute events over that time.

\section{Disclosure}

The authors declare no conflicts of interest in this work. No financial support was received by the authors for the preparation of this paper.

\section{References}

1. Ozben B, Ekmekci A, Bugra Z, Umman S, Meric M. Multiple coronary thrombosis and stent implantation to the subtotally occluded right renal artery in a patient with essential thrombocytosis: a case report with review. J Thromb Thrombolysis. 2006;22(1):79-84.

2. Elliott MA, Tefferi A. Thrombosis and haemorrhage in polycythaemia vera and essential thrombocythaemia. Br J Haematol. 2005;128(3): 275-290.

3. Pande S, Joshi R, Pande R. Essential thrombocythemia in a young man treated for myocardial infarction. BMJ Case Rep. 2010;2010.

4. Rossi C, Randi ML, Zerbinati P, Rinaldi V, Girolami A. Acute coronary disease in essential thrombocythemia and polycythemia vera. $J$ Intern Med. 1998;244(1):49-53.

5. Mizuta E, Takeda S, Sasaki N, et al. Acute myocardial infarction in a patient with essential thrombocythemia: successful treatment with percutaneous transluminal coronary recanalization. Circ J. 2005;69(8): $1000-1002$.

6. Leng S, Nallamothu BK, Saint S, Appleman LJ, Bump GM. Clinical problem-solving. Simple and complex. N Engl J Med. 2013;368(1): $65-71$.

7. Fuster V, Badimon L, Badimon JJ, Chesebro JH. The pathogenesis of coronary artery disease and the acute coronary syndromes (1). $N$ Engl J Med. 1992;326(4):242-250.

8. Douste-Blazy P, Taudou MJ, Delay M, et al. Essential thrombocythaemia and recurrent myocardial infarction. Lancet. 1984;2(8409):992.

9. Bildirici U, Celikyurt U, Ural E. Essential thrombocythemia: a case of acute ST-segment elevation myocardial infarction in a young female. Clin Cardiol. 2009;32(2):104-105.

10. Barbui T, Finazzi G. When and how to treat essential thrombocythemia. N Engl J Med. 2005;353(1):85-86.

11. Kumar V, Abbas AK, Fausto N, Mitchell R. Robbins Basic Pathology. 8th ed. Philadelphia, PA: Saunders Elsevier; 2007.

12. Harrison CN, Campbell PJ, Buck G, et al; United Kingdom Medical Research Council Primary Thrombocythemia 1 Study. Hydroxyurea compared with anagrelide in high-risk essential thrombocythemia. N Engl J Med. 2005;353(1):33-45.

13. Watanabe T, Fujinaga H, Ikeda Y, et al. Acute myocardial infarction in a patient with essential thrombocythemia who underwent successful stenting: A case report. Angiology 2005;56(6):771-774.

14. Henriques JP, Zijlstra F, Ottervanger JP, et al. Incidence and clinical significance of distal embolization during primary angioplasty for acute myocardial infarction. Eur Heart J. 2002;23(14):1112-1117.

15. Kumagai N, Mitsutake R, Miura S, et al. Acute coronary syndrome associated with essential thrombocythemia. J Cardiol. 2009;54(3): 485-489.

16. Tefferi A, Fonseca R, Pereira DL, Hoagland HC. A long-term retrospective study of young women with essential thrombocythemia. Mayo Clin Proc. 2001;76(1):22-28.

17. Landolfi R, Di Gennaro L. Prevention of thrombosis in polycythemia vera and essential thrombocythemia. Haematologica. 2008;93(3): 331-335.

18. Cohen M, Diez J, Levine GN, Ferguson JJ III, Morrow DA, Rao SV, Zidar JP. Pharmacoinvasive management of acute coronary syndrome: incorporating the 2007 ACC/AHA guidelines: the CATH (cardiac catheterization and antithrombotic therapy in the hospital) Clinical Consensus Panel Report-III. J Invasive Cardiol. 2007 Dec;19(12):525538; quiz 539-540. 
Journal of Blood Medicine

\section{Publish your work in this journal}

The Journal of Blood Medicine is an international, peer-reviewed, open access, online journal publishing laboratory, experimental and clinical aspects of all topics pertaining to blood based medicine including but not limited to: Transfusion Medicine; Blood collection, Donor issues, Transmittable diseases, and Blood banking logistics; Immunohematology; Artificial and alternative

blood based therapeutics; Hematology; Biotechnology/nanotechnology of blood related medicine; Legal aspects of blood medicine; Historical perspectives. The manuscript management system is completely online and includes a very quick and fair peer-review system. Visit http://www.dovepress.com/ testimonials.php to read real quotes from published authors.

Submit your manuscript here: http://www.dovepress.com/Journal-of-blood-medicine-journal 